\title{
A Median Filter With Evaluating of Temporal Ultrasound Image for Impulse Noise Removal for Kidney Diagnosis
}

\author{
Rasheed Rebar Ihsan ${ }^{1, *}$, Saman M. Almufti 2, Ridwan B. Marqas ${ }^{3}$ \\ ${ }^{1}$ Department of Information Technology, Duhok Private Technical Institute Duhok, Iraq, rasheed.sarky@gmail.com \\ 2 Department of Computer Science, Nawroz University Duhok, Iraq, saman.Almofty@nawroz.edu.krd \\ ${ }^{3}$ Computer Engineering, Firat University, Elazig, Turkey, 191129206@firat.edu.tr \\ *Correspondence: rasheed.sarky@gmail.com
}

\begin{abstract}
Ultrasound imaging helps the doctor to view the tissues and organs in the body's abdominal area with no ionization risks compared to other internal organ examination methods dependent on radiation. It offers highly precise renal imaging of suspected acute kidney diseases. This paper proposes temporary filtering methods to improve ultrasound images from ultrasonic kidney video. The proposed filters focus on the detection and diagnosis of kidney disease by processing consecutive images of the acquired kidney video. Extending the spatial median image filters to temporal dimensions after the picture frames are manually clipped and aligned in MATLAB by image processing Toolbox to suppress speckle noise, and enhance a doctor's diagnostic information quality.
\end{abstract}

Keywords: Ultrasound Kidney Image, Image Enhancement by Video, Temporal Median Filter

Received: April 19, 2020 /Accepted: May 21, 2020 / Online: May 22, 2020

\section{INTRODUCTION}

Doctors use ultrasonic images very frequently for diagnosis. In certain instances, certain photos are not precise enough to distinguish particular structures and formations. Raw ultrasonic images require operations for acquisition artefact correction and noise filtering to improve analysis quality.

Medical Ultrasound (US) imaging has recently been broadly used in diagnostic applications for illness due to its low cost, non-invasive design, protection, portability and real-time capability [1,2]. Several diagnostic diseases of human organs such as chest, kidney, abdomen, back, breast, heart, coronary artery, abdominal cavity, and scrotum exist [3].

The images produced from ultrasound imaging, however, are of relatively low quality due to the presence of speckle noise or multiplicative noise that instigates granular effect on the image [4]. In order to, there is also the speckle noise and the random Gaussian additive noise in the US photos. The presence of these kinds of noises prevents the precision of the clinical diagnosis by reducing the quality of vision. In addition, diagnosis that consumes more time and post-processing, such as segmentation and extraction of data, is becoming very difficult
[5]. Noise reduction or denouncement of US images therefore becomes a necessary necessity without the impact of edges and textures in the images. Therefore, image processing methods are required in order to improve the quality of the kidney ultrasound image. There are many image processing techniques developed to enhance ultrasound image to get clearer and higher quality image by reducing the noise in the image [6]

Median filtering is proposed to reduce the speckle noise in medical US images. This filtering by replacing the pixel value in the mask with a mean value (order statistics), the median filter eliminates noise. Hence, in homogenous areas, this median filtering scheme exhibits excellent noise removal efficiency; however, it has poor edge preservation efficiency at the edges.

This filter sorts the pixel values in the neighborhood of the target pixel, and decides the value at the middle of the sorted list for pixel value of the center element [7].

There are numerous earlier studies conducted in contrasting various US noise repression and image enhancement tools for US images. However, they are focused only on enhancement of a single image frame. The proposed temporal filtering provides enhancement of an image in a video record, enriching the 
information contents of a single frame by including extra information from consequent images, while old methods provide only a smoothening effect that leads to information loss. Medical Ultrasound imaging is also known as diagnostic sonography or Ultrasonography and it has become one of the main techniques in today's medical diagnosis. It is inexpensive, widely accessible and comparatively safe to the users as well as the operators. A sonographer's task is to collect reliable sonographic documents under supervision of a physician to diagnose a diversity of circumstances and illnesses. The proposed technique can be adopted into the standard processes of diagnostic sonography for the medical benefits of the human being.

\section{LITERATURE REVIEW}

Removing noise from the original image remains a difficult study in the processing of images. There is generally no specific noise-reduction enhancement solution. Several methods were adopted, each with its own definitions, benefits and disadvantages. The speckle noise is usually seen in medical images with ultrasound. There are a number of studies conducted in the past, which compare various ultrasound speckle suppression and image enhancement methods for US images.

Donoho presented a soft-thresholding de-noising method in which the image observed is decomposed into wavelet domain. Using Donoho's method, Thakur et al made a comparative study of different wavelet filters that have various thresholding values of US images and have come to the conclusion that de-noising methods of this kind are effective in that they preserve, besides suppressing the noise, the edge details [8]. Local histogram equalization (LHE) has been widely used. The region of interest in medical ultrasound image is usually surrounded by large areas of dark background, which include modest information but consumes a huge amount of computational resource. Direct use of LHE will not only consumes time but will in addition affect the global visibility due to the distortion of the background. The LHE algorithm is used on the sub-block's center pixel. The technique will speed up processing frequency and significantly decrease noise distortion.

Li \& Liu in their research used dynamic filtering techniques particularly for image enhancement [9]. Gajanand Gupta compared the improved technique of median filter to median and mean filter techniques [5]. The median filter is among the most widely used nonlinear spatial domain filters. In 2010 the scientist Yen suggested the use of ultrasound enhancement method by employing the dual tree wavelet method [10]. Ultrasound scanning is highly open to human error while measuring size of organs and tissues because of noisy US images and inexperienced sonographers. Filtering the noise by median filter reduce the effect of noise, but it also reduces information content of the images. The proposed filters can help to sonographer to develop better filtered images with higher information contents such as better edges, correct contrast, etc.

Birla in 2015 implemented a median filter which is a special case of nonlinear filters that are used to smooth signals. Their median filter is now commonly used to reduce noise and smooth out the images [11]. In 2011 Supriyanto used topological median filters to boost conventional median filter which is designed to retain sharp edge [12]. While Tukey proposed an image denoising algorithm based on average filter with maximization and minimization for region smoothness, unidirectional Median filter for edge region, and median filter for indefinite area [13]. It was noticed that when both Gaussian and impulse noises corrupt the image, due to their algorithm, neither Average filter nor Median filter algorithm would obtain a result sufficiently good for filtering the noises. Hakan in 2002 has introduced an improved adaptive median filtering system to oppose impulse noise reduction [14]. Dhanasekaran showed an adaptive median filter (AMF) as the best filter to eliminate salt and pepper noise from image sensing [15].

\section{MEdicAl IMAgE ENHANCEMENT TECHNIQUES}

There are many techniques used to enhance the medical image most of them are listed below:

A. Image Registration or Alignment is used to coordinate the representations of tissues or organs with each other to match exactly [16]. When images are taken from various angles and at different times, sometimes with separate instruments or sensors, these images are merged by image capture. That process is called registration of images. Image recording contrasts a reference image with the secondary images in A geometrical form. Medically, CT and NMR are used to integrate the image details to provide more accurate images of the tissues. It helps to diagnose and track tumor development, perform treatment, and compare patient data with anatomical atlases, especially in cartography and computer vision, that is to say, targeting localization, automated quality control among others [17].

$B$. Image segmentation uses a region's color to distinguish it, which may correspond to a picture of a tissue or organ. It removes any irregular clot / lesion or presence of blood vessels / cells in an image [18]. It is one of the major medical image processing procedures which is usually performed after enhancement. The regions have similar aspects on features in a segmented picture. For example, the property difference on the grey scale, structure or color from one region to another. This divides the picture into classes corresponding to objects across the viewing range, which are key in identifying otherwise dissimilar forms of leukocytes resulting in the severity of the tumor or blood vessels or any other problems occurring in the human body [19].

C. Boundary detection is an important operation in the processing of medical images. It detects the image's structural details and thus significantly decreases the data to be treated [20]. Grey scale density varies, while edge is present. Possibly edge image labelling as gradient image. The picture boundaries help to define the location and the dimension of the objects for example, a structurally 
irregular lesion, tumor, blood cell or vessel. The medical photos aren't identical due to lighting and contrast problems. As such, the boundaries are normally strengthened at first by applying the method of edge detection.

D. Morphology operations are nonlinear methods of image processing to reshape the region inside an image. We deal with picture type features such as dilution, thickening, etc. [21]. It is most often important to collect an image skeleton or to erode / dilate an image, or to open / close an image to delete or plug small gaps or holes in an image shape.

\section{PROPOSED FILTER}

Image pre-processing is applied to raw images in order to reduce image noise. Images with speckle noise, reflection and similar factors are pre-processed to reduce the effects of these factors. Filtering is among the pre-processing techniques which help in enhancing the images by removing the noise of the images [9].

Filtering techniques decrease undesirable signals which are recognized as "noise". Efficiency of a filter measures the reduction of undesirable signals while keeping desired signal. Image enhancement and filtering methods are applied on the image before the main operation such as object detection in order to reduce the degrading interference of the impulse noise in the image. Impulse noise was mostly caused when signal is converted from analogue to digital as the analogue-to-digital conversion error. For best efficiency, the type of filter to be applied on an image depends on the noise and intensity of the noise [22]. The median filter is used in this study:

\section{A. Median Filter}

This most common technique used for the reduction of noise. It is a technique of "non-linear" filtering. This is used to suppress the grayscale picture of "salt and pepper noise" [23]. Median filter is based on pixel mean. Median filter benefits in reducing salt and pepper noise and Speckle noise are effective. The edges and boundaries are also conserved. as shown in Fig. 1. The median filter performs better than the other techniques, such as mean filtering, for preserving information in the image [9]. The key disadvantages are complexity and time consumption [24].

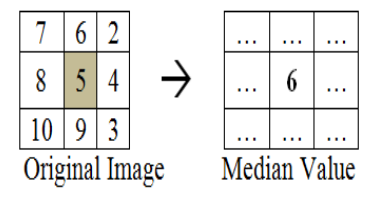

Sorted values: $2,3,4,5,6,7,8,9,10$; value of middle item: 6

Fig.1. Calculating the Median Value of a Pixel Neighborhood

\section{MEdiCAL IMAGE NOISE}

Due to a variety of non-idealities in the imaging process, medical images are often compromised by impulsive, additive or multiplicative noises. The noise normally corrupts medical images by replacing some of the original pixels with new pixels with luminance values similar to or equal to the minimum or maximum of the dynamic luminance range allowed. In the medical picture, the identification of the type of noise is done in two stages. In the first point, the presence of the impulsive noise is detected using a criterion. If the result of this criterion is negative, the image shall then be submitted to the second stage of another criterion to classify either the additive or the multiplicative nature of the noise [25].

\section{TYPES OF NOISE}

A. Impulsive Noise (Salt \& Pepper) The noise of the salt-andpepper type is generally caused through errors in the data transfer of malfunctioning pixel elements in camera sensors, incorrect memory positions or timing errors in the digitization process.

B. Additive Noise (Gaussian) The Gaussian noise is quite commonly used to model normal noise processes, like those that happen in the image acquisition system from electronic noise.

C. Multiplicative noise (Speckle) Speckle noise is a ubiquitous object in the image that prevents the perception of medical image's optical specificity. This type of noise is very common and corrupted the medical image. This paper proposes median filtering techniques based on doctor's assessment methods for the removal of speckle noise. A number of success full experiments validate the proposed filtering model [26].

\section{PROPOSED FILTERS AND METHODOLOGY}

This study measures image enhancement with respect to a diagnosis by applying one specific image filtering method to resulting images of ultrasound videos in the kidney. A video consists of a series of picture frames to display for a fraction of seconds. The human eyes incorporate the series of images into a continuous motion. A video signal's most common raw format is RGB analogy component video, where the images are scanned in a row for all three-color components Concurrently.

MATLAB provides a toolbox for image processing to collect frames from any complex multimedia video format (i.e., MPEG, AVI etc.) into RGB photos.

An RGB image is a three-matrix representation of a single frame from a video. The image $f$ includes the pixel values red, green and blue $f(x, y, c)$, where $c$ is the color index, and $x, y$ are pixel co-ordinates.

A grayscale image is a representation by a single matrix of a grey scale picture, $\mathrm{f}$. The coordinate pixels $\mathrm{f}(\mathrm{x}, \mathrm{y})$ in range $(0$, 1 ), where 0 corresponds to black, and 1 is white. This study is 
only concerned with grayscale videos, since ultra-sonographic devices provide only a raw source of grayscale footage.

A raw video is a video directly taken from a video source, such as a video camera, a radar screen, or an ultrasonography device. The video source noise requires filtering relative to the relevant picture signal, to eliminate the noise.

An image filter is a mathematical operation on a raw image's pixel values to remove the image's noise without reducing the meaningful part of the image. Therefore, a temporal image filter is a mathematical process that transforms a series of video frames into a single image with less noise than any single video source frame as shown in Eq. (1).

$$
D(i, j)=\sum_{\substack{p, q=-s, \ldots, s \\ r=-t, \ldots, t}} h(p, q, r) \frac{d(p+i, q+j, r+F)}{(1+2 s)^{2}(1+2 t)}
$$

Where: $D(i, j)$ is the intensity of the pixel at coordinate $i, j$ of the filter output; di,j,r is the pixel intensity of the pixel at coordinate $\mathrm{i}, \mathrm{j}$ of the $\mathrm{r}^{\text {th }}$ raw image in the video; $\mathrm{S}$ is the spatial half-depth, $\mathrm{T}$ is the temporal A temporal median filter is calculated by the median operation along the pixels in the neighborhoods of $\mathrm{s}$ and $t$ around the center pixel $(i, j)$ as Eq. $(2)$ :

$$
D(i, j)=\operatorname{median}_{\substack{p=i-s, \ldots, i+s \\ q=j-s, \ldots, j+s \\ r=k-t, \ldots, k+t}}\{d(p, q, r)\}
$$

It is possible to have only spatial filtering effect by setting $\mathrm{t}=0$, as well as, it is possible to obtain a purely temporal median filter by setting $\mathrm{s}=0$, but $\mathrm{t} \neq 0$.

The proposed temporal filters have both spatial and temporal smoothening effects as they translate the pixel intensity to the values in the pixel's spatial and temporal neighborhoods between the maximum and minimum intensities, making them strong candidates for suppressing the impulse noise in the images. To take one example, Fig. 2 displays the raw center image and the corresponding strictly temporary filtered median image at half-depth $s=0, t=9$. It is obviously less noisy and rich in data, making it suitable for diagnostic purposes to medical experts

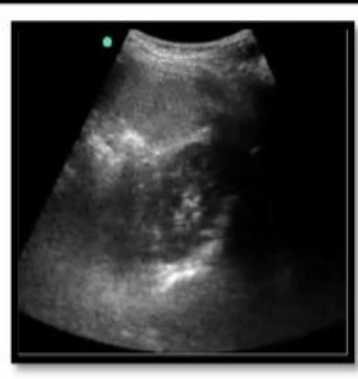

Original Frame

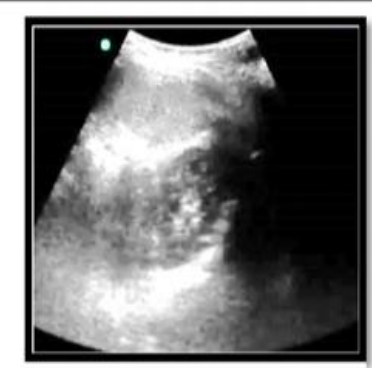

Filtered Frame by using Median Filter
Fig. 2. Pre-Processed and Filtered Image of Kidney

\section{A. Determination of the Best Temporal Filter For Kidney Ultrasound}

Fig. 3 displays the flow diagram of the optimization process to decide the best temporal filter, and its parameters. A collection of kidney-ultrasound video avi format was captured and saved in MPEG format in this study. The video image frames were recorded in MATLAB using the phase function of the image processing toolbox. The photos are cropped and registered in order to overlap the photos of the kidneys to the same coordinates on each image.

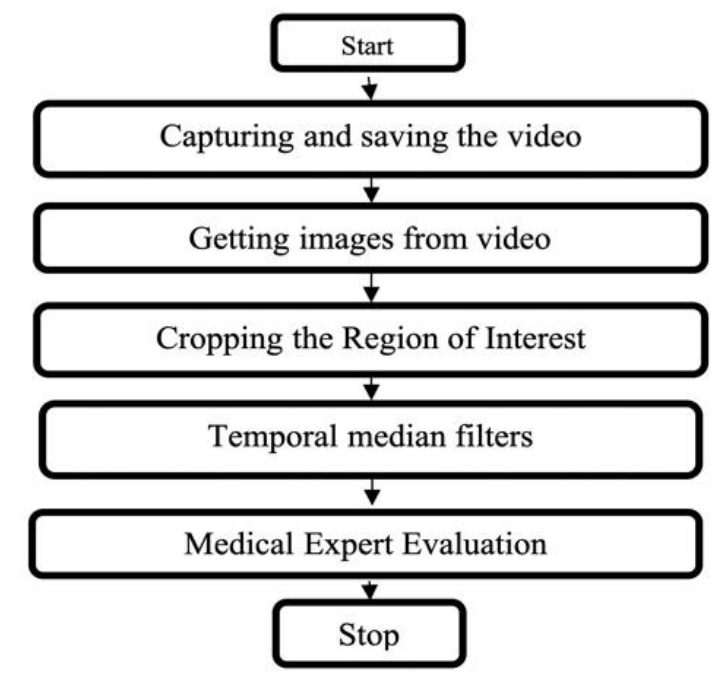

Fig. 3. Overview of Assessment Process

Throughout the evaluation process, a total of 11 videos are processed by means of median strictly temporary filters at various temporal half-depths from 0 to 5 , where 0 corresponds to using only one image input, and 5 corresponds to using 11 frames to produce one filtered image output. For examples, the suggested filter uses previous 2 frames, plus the third frame as the center frame, plus the next two frames in temporal mask filter operation.

During patient evaluation, video recording is performed by medical doctors and expert technicians using an ultrasonic unit. All 12 videos are taken with the same camera, but from different patients, this is determined by the physician considering the importance of the diagnostic images in the USA. Nevertheless, similar advantages of temporal filtering are obviously expected to be observed on other US system brands including 2D, 3D, 4D, $5 \mathrm{D}$ and Doppler Ultrasound.

These 12 video samples are made up of 6 normal kidney videos, 6 kidney calculi (kidney stones, and complex cysts). They are around 20 -second clips with 25 frames per second in avi-file format. As a result, they will record 25 frames per second.

One of them was later identified as unfit for treatment, and the remaining 11 videos are analyzed to choose the best filter to improve video captures to an image that is subject to treatment 
of kidney disease. Getting Consecutive Images of a Video is carried out after a medical expert agrees on each video's significant frame to assess the $\mathrm{F}$ center frame for temporal filtering. Fig. 4 displays an initial picture of a kidney video in the middle.

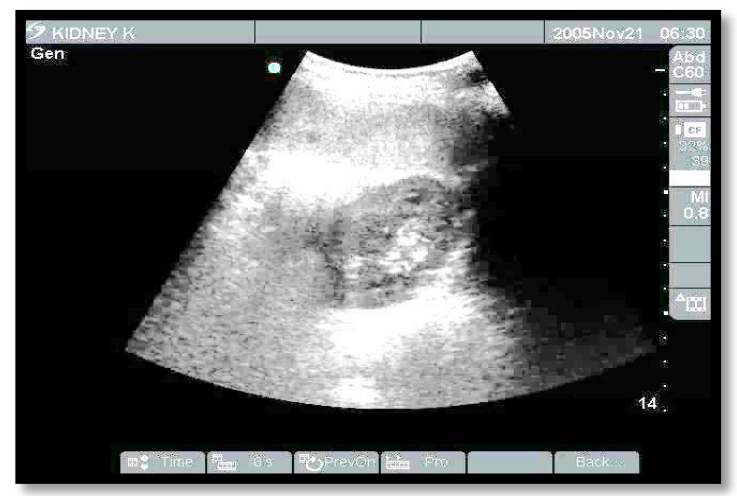

Fig.4. The Centre Image of a Kidney Ultrasound Video

So previous five, and next five frames starting from Fth frame are recorded in grayscale image format, which MATLAB saves in a two-dimensional matrix. To record the frames for the location and orientation of the photos of the kidneys, you need to crop the area of interest. This move is carried out manually with the aid of an image editor. Cropping eliminates the majority of the black areas around the rein, and only that section remains in the cropped picture for diagnosis.

On the manually cropped and aligned collection of images, temporal median and filters are applied to create a set of filtered images of the kidney This method applies the filters individually on all 11 frames. Medical Expert Evaluation is the final step in determining the best filtering method.

All images produced from a single video capture are guided to one U.S. expert, so that after an interview, a total of 11 medical experts blindly analyzed a set of filtered images to notify them about the filtering process used in image production. The assessment is carried out by assigning a score for what image, without knowing which picture the filter generates, and finally they are asked to fill in feedback on the best and worst of the diagnostic images.

Kidney screening is used by US physicians to determine the length and width of the kidney. For this procedure clear edges of the picture of the kidney are essentially required for a more precise measurement.

\section{B. Implementation of Temporal Image Filtering}

As stated in the previous segment, the experts have selected the most significant image of the videos to assess K's importance. Then, videos were stripped off of all unwanted frames to include just 11 frames, from 5 frames before the Kth frame to 5 frames after the Kth frame. A description of the image selected and strictly spatial filtered images are shown in the Fig. 5. The MATLAB script which has been created loads a video by its file name into memory. The MATLAB script pre-processing segment succeeds in capturing 11 frames from the loaded file.
The photos are ready for temporal filtering after manual entry of the cropping box corners.

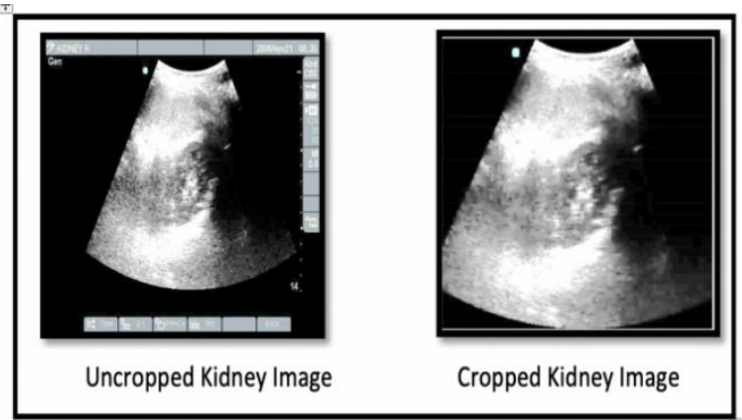

Fig. 5. Cropping and Aligning the Image Frames

The next MATLAB script segment includes the temporal filters, these are applied in a for-to the appropriate images, increasing the depth of the temporal filter median. The filtered images are printed in $2 \times 2$ subplot matrix as shown in Fig. 6 , where (1) the top-left cell contains the original frame, (3) the bottom-left cell has ( $3 \times 3)$-medium-filtered image, for the spatial half-depths from 0 (raw image, Frame $=1$ label) to 5 (frame $=6$ labelling).

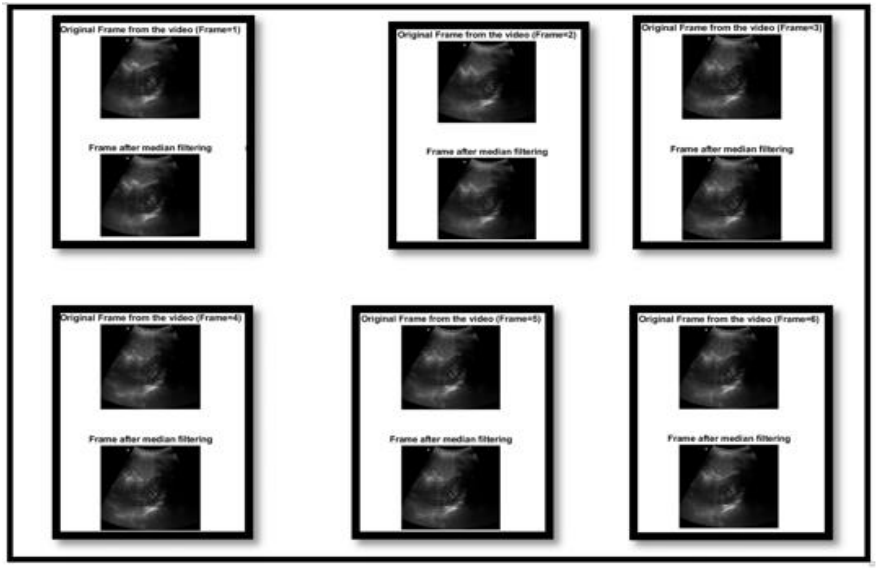

Fig. 6. A Sample of Diagnostically Significant Image and its $3 \times 3$ Median Images

\section{Evaluation process of tempora filters}

In the assessment process a single medical expert analyzed 18 filtered images from each recording. The score is required to rate each image's significance in a scale of 1 to 10. Experts were asked to fill in comments for the pictures along with a ranking. These findings are set out in Table I.

TABLE I : DOCTORS COMMENTS ON MEDIAN FILTER

\begin{tabular}{|l|l|}
\hline \multicolumn{1}{|c|}{$\begin{array}{c}\text { Names of } \\
\text { Doctors }\end{array}$} & \multicolumn{1}{c|}{ Comments to Median Filter } \\
\hline $\begin{array}{l}\text { Dr.Shirwan } \\
\text { S. Atroshy }\end{array}$ & $\begin{array}{l}\text { Regarding the second filtration, which is the median } \\
\text { filtration, regarded the best between the three and we can } \\
\text { depend even till the first sixth. However even this; the last } \\
\text { three are terrible in resolution and it's difficult to depend } \\
\text { on it. }\end{array}$ \\
\hline
\end{tabular}




\begin{tabular}{|l|l|}
\hline $\begin{array}{l}\text { Dr.Zhivan } \\
\text { F. Amidy }\end{array}$ & Median filter shows good contrast of tissue and clear edges. \\
\hline $\begin{array}{l}\text { Dr.Jalal J. } \\
\text { Zanganna }\end{array}$ & $\begin{array}{l}\text { The median filter is better showing the details of the } \\
\text { calyceal system of the kidney. }\end{array}$ \\
\hline $\begin{array}{l}\text { Dr.Shilan } \\
\text { M. Karkuki }\end{array}$ & $\begin{array}{l}\text { After using median filter, the noise in the pictures are much } \\
\text { reduced, becoming much better quality and good resolution } \\
\text { without effecting the fine details. }\end{array}$ \\
\hline $\begin{array}{l}\text { Dr.Hero O. } \\
\text { Sindy }\end{array}$ & $\begin{array}{l}\text { In this type of filter ("Median filter") the resolutions of the } \\
\text { Kidney clearer than the previous filter ("Mean Filter"). }\end{array}$ \\
\hline $\begin{array}{l}\text { Dr. Süreyya } \\
\text { Vudali } \\
\text { Doğruyol }\end{array}$ & $\begin{array}{l}\text { Above all of the filters median filter has best resolution in } \\
\text { my opinion. }\end{array}$ \\
\hline
\end{tabular}

\section{Questioner Format of Expert Assessment}

Samples of video-4 expert scores are listed in Table II through 4.9. We also offer hint about how assessment forms are organized. The experts openly rated each image according to single criteria: the image's sufficiency for diagnosing kidney disease, and for correct tissue measurements and any formations. Though the photos are somewhat close, for example, the images are quite similar. Once again expert in Table II considered temporal median filtering with depth 5 to be better than depth 3 instances. The scores above 7 basically means that all images are highly appropriate for diagnosis, but temporary median filtering at depth 5 rendered the image better.

TABLE II: EXPERT SCORES FOR VIDEO CLIP 4 WITH MEDIAN FILTER

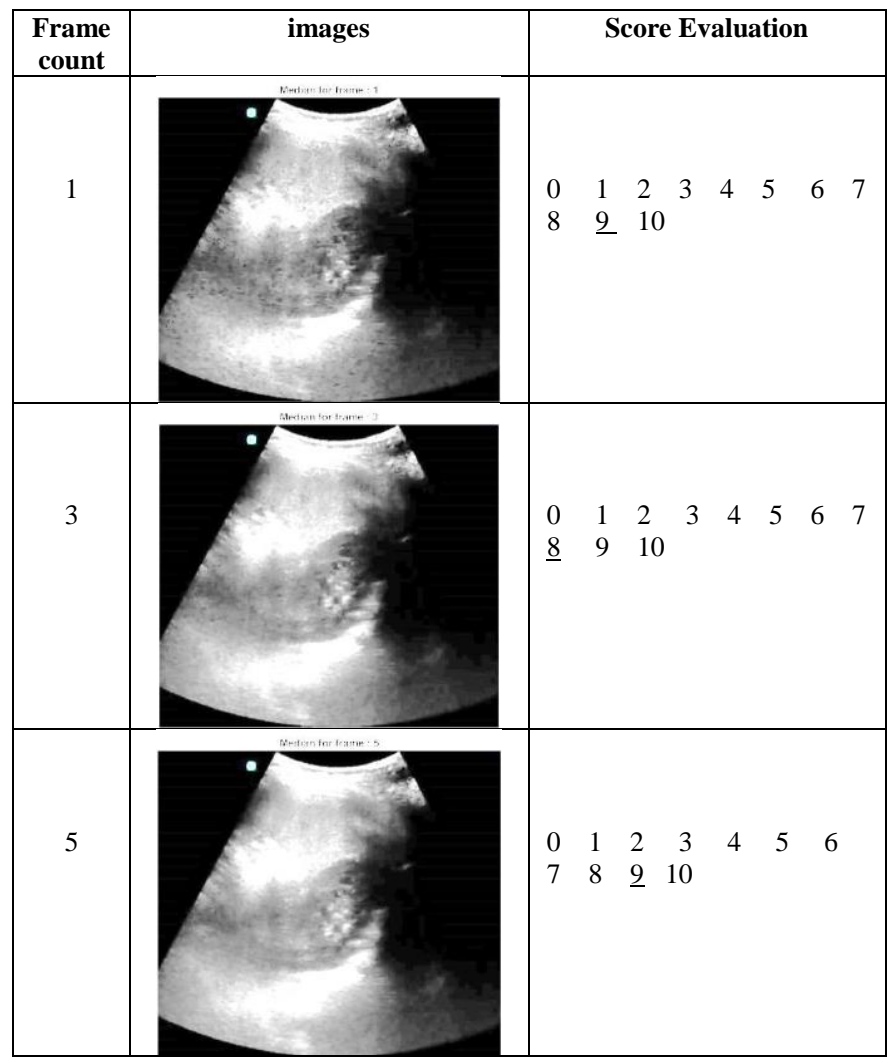

Following the expert assessments for the 18 images of each of the 12 video sources, the scores determined by experts for each image are filled in Table III, video 12 is collected in the table with very low scores as planned, because According to expert opinion, the content of the video was not considered to be appropriate for any diagnostic or measurement function.

In the table III, to determine the median filter, and the best temporal depth of the filter, the sum of all images was evaluated for the same type for both the same type and the same depth. The aggregate total over 12 video sources for all depths of the same sort indicates that the $(78-28) / 28=1.78 \%$ for the median filter when temporal depth is set to 11 frames. It is determined, as a result of these tests, that temporal filtering is extremely useful in enhancing ultrasound images. Filtering the raw image with a temporal depth median of 11 frames produces a nearly threefold cleaner US image compared to the spatially filtered images. Finally, based on the doctor's evaluation, the efficiency of the filters is compared.

TABLE III: EXPERT ASSESSMENT SCORES FOR IMAGES OF ALL TESTED FILTERS

\begin{tabular}{|c|c|c|c|c|c|c|}
\hline Filter: & \multicolumn{7}{|c|}{ Temporal Median } \\
\hline $\begin{array}{c}\text { Depth: } \\
\text { Video }\end{array}$ & $\mathbf{1}$ & $\mathbf{3}$ & $\mathbf{5}$ & $\mathbf{7}$ & $\mathbf{9}$ & $\mathbf{1 1}$ \\
\hline Video 1 & 2 & 3 & 4 & 5 & 7 & 8 \\
\hline Video 2 & 8 & 7 & 6 & 6 & 4 & 4 \\
\hline Video 3 & 1 & 2 & 3 & 6 & 8 & 9 \\
\hline Video 4 & 9 & 8 & 9 & 7 & 5 & 3 \\
\hline Video 5 & 1 & 1 & 1 & 3 & 4 & 5 \\
\hline Video 6 & 2 & 3 & 3 & 3 & 4 & 4 \\
\hline Video 7 & 2 & 3 & 5 & 6 & 8 & 8 \\
\hline Video 8 & 0 & 1 & 3 & 5 & 6 & 8 \\
\hline Video 9 & 0 & 2 & 3 & 5 & 7 & 9 \\
\hline Video 10 & 0 & 3 & 4 & 6 & 7 & 9 \\
\hline Video 11 & 2 & 3 & 4 & 5 & 7 & 8 \\
\hline Video 12 & 1 & 2 & 3 & 2 & 3 & 3 \\
\hline Sum & $\mathbf{2 8}$ & $\mathbf{3 8}$ & $\mathbf{4 8}$ & $\mathbf{5 9}$ & $\mathbf{7 0}$ & $\mathbf{7 8}$ \\
\hline Overall & & & & $\mathbf{3 2 1}$ & & \\
\hline Sum & & & & & & \\
\hline
\end{tabular}

\section{CONCLUSION}

A new image enhancement technique on $b$ medical ultrasonic kidney imaging based on median filters has been proposed in this paper. By extending the filtration techniques to the temporal domain, the method improves the image by processing the consecutive frames of a video. The suggested approaches are tested by doctor's assessment on twelve video clips of the kidney diagnosis. The images are divided between a total of 6 doctors in radiology to rate them above 10, a higher score meaning the image is more valuable for diagnostic purposes. We have also highlighted the best among a collection of filtered pictures. From this evaluation it is deduced that the temporal median filter with depth of 11 frames provides maximum diagnostic improvement, keeping the image information obvious and remarkable while eliminating noise considering the diagnostic purpose.

\section{REFERENCES}

[1] Ramos-Llorden G, Vegas-Sanchez-Ferrero G MartinFernandez M, Alberola-Lopez C, Aja-Fernandez S. Anisotropic diffusion filter with memory based on speckle statistics for ultrasound images. IEEE Trans Image Proc 2015; 24: 345-358. 
[2] Sivakumar R, Gayathri MK, Nedumaran D. Speckle filtering of ultrasound B-scan images-a comparative study between spatial and diffusion filters. International conference on Open Systems (ICOS), IEEE Conference 2010; 80-85.

[3] Ramos-Llorden G, Vegas-Sanchez-Ferrero G, MartinFernandez M, Alberola-Lopez C, Aja-Fernandez S. Fast anisotropic speckle filter for ultrasound medical images. Mediterranean 13th Conference on Medical and Biological Engineering and Computing Springer Cham 2013; 253-256.

[4] Bhateja V, Singh G, Srivastava A. A novel weighted diffusion filtering approach for speckle suppression in ultrasound images. Proceedings of the International Conference on Frontiers of Intelligent Computing: Theory and Applications (FICTA) Springer Cham 2013; 459-466.

[5] Gupta D, Anand RS, Tyagi B. Speckle filtering of ultrasound images using a modified non-linear diffusion model in nonsubsampled shearlet domain. IET Image Proc 2014; 9: 107-117.

[6] Htwe, Kinsana, \& Aung. S. Kh. (2011). "Classification Of Appendicitis Based On Ultrasound Image" of the $15^{\text {th }}$ WSEAS International Conference on Computers, World Scientific and Engineering Academy and Society (WSEAS): Corfu Island, Greece, pp. 223-227.

[7] Gnanadurai, D., Sadasivam, V., Nishandh, J.P.T., Muthukumaran, L. and Annamalai, C., 2009. Undecimated double density wavelet transform based speckle reduction in SAR images. Computers \& Electrical Engineering, 35(1), pp.209-217.

[8] Wan, M. H., \& Supriyanto, E. (2011). Comparative evaluation of ultrasound kidney image enhancement techniques. International Journal of Computer Applications, 21(7), 15-19.

[9] Li, X., \& Liu, D. C. (2007, August). Ultrasound image enhancement using dynamic filtering. In Fourth International Conference on Image and Graphics (ICIG 2007) (pp. 106-109). IEEE.

[10] Yen, W. C., \& Tai, S. C. (2010, October). Dual-tree wavelet based algorithm for speckle reduction and edge enhancement in ultrasound images. In 2010 3rd International Conference on Biomedical Engineering and Informatics (Vol. 1, pp. 208-212). IEEE.

[11] Birla, A., \& Shantaiya, S. (2015).Performance Analysis of Image Enhancement Techniques for Brain Tumor Images. International Journal of Emerging Trends \& Technology in Computer Science (IJETTCS). Volume 4, Issue 5(2).India.

[12] Supriyanto, E., Tahir, N. A., Nooh, S. M., Arooi, A., \& Hafizah, W. (2011, July). Automatic Ultrasound Kidney's Centroid Detection System. In WSEAS International Conference on Computers (ISI and SCOPUS Indexed), Greece.

[13] J. W. Tukey. 1974. Nonlinear (nonsuperposable) methods for smoothing data. In: Proc. Congr. Rec. EASCOM '74. pp. 673681.

[14] Hakan Güray Senel, Richard Alan Peters and Benoit Dawant. 2002. Topological Median Filter. IEEE Trans on Image Processing. 11(2): 89-104.

[15] Dhanasekaran, D., Krishnamurthy, A., \& Ramkumar, J. (2009, January). High speed pipeline architecture for adaptive median filter. In Proceedings of the International Conference on Advances in Computing, Communication and Control (pp. 597600).

[16] Yanchun Wang, Dequn Liang, Heng Ma and Yan Wang. 2006. An Algorithm for Image Denoising Based on Mixed Filter. Proceedings of the 6th World Congress on Intelligent Control and Automation. June 21-23. pp. 9690-9693.

[17] Mamta Juneja and Rajni Mohana. 2009. An Improved Adaptiv Median Filtering Method for Impulse Noise Detection. International Journal of Recent Trends in Engineering. 1(1): 274278.

[18] Szabo, T. L. (2004). Diagnostic ultrasound imaging: inside out. United Kingdom, UK: Academic Press.

[19] Supriyanto, E. (2011). Segmentation of prostate tumor for gamma image using region growing method, in Proceedings of the 15th WSEAS international conference on Computers. World Scientific and Engineering Academy and Society (WSEAS): Corfu Island, Greece. p. 189-194.
[20] Zitova, B., \& Flusser, J. (2003). Image registration methods: a survey. Image and vision computing, 21(11), 977-1000.

[21] Htwe, Kinsana, \& Aung, S. Kh. (2011). "Classification Of Appendicitis Based On Ultrasound Image" of the $15^{\text {th }}$ [WSEAS]International Conference on Computers, World Scientific and ]]Engineering Academy and Society (WSEAS): Corfu Island, Greece, pp. 223-227.

[22] Chaira T. (2015). Medical Image Processing, in Medical Image Processing Advanced Fuzzy Set Theoretic Techniques, New York, NY.CRC Press, pp. 23-25.

[23] Achim, A., Bezerianos, A., \& Tsakalides, P. (2001). Novel Bayesian multiscale method for speckle removal in medical ultrasound images. IEEE transactions on medical imaging, 20(8), 772-783.

[24] Rajesh C. patil, A.S. Bhalchandra, "Brain tumor extraction from MRI images Using MAT Lab", IJECSCSE, ISSN: 2277-9477, Volume 2, issue 1

[25] Gopal,N.N. Karnan, M. , -Diagnose brain tumor through MRI using image processing clustering algorithms such as Fuzzy C Means along with intelligent optimization techniques Page(s): 1 - 4, Computational Intelligence and Computing Research (ICCIC), 2010 IEEE International Conference, 28-29 Dec. 2010. Bin-Habtoor, A.S.Y. and Al-amri, S.S., 2016. Removal speckle noise from medical image using image processing techniques. International Journal of Computer Science and Information Technologies, 7(1), pp.375-377. 